\title{
Unified Derivation of Bohmian Methods and the Incorporation of Interference Effects ${ }^{\dagger}$
}

\author{
Yair Goldfarb, $*, \star$ Jeremy Schiff, $\$,, \|$ and David J. Tannor \\ Department of Chemical Physics and Department of Mathematics, The Weizmann Institute of Science, \\ Rehovot, 76100, Israel
}

Received: April 29, 2007; In Final Form: June 18, 2007

\begin{abstract}
We present a unified derivation of Bohmian methods that serves as a common starting point for the derivative propagation method (DPM), Bohmian mechanics with complex action (BOMCA), and the zero-velocity complex action method (ZEVCA). The unified derivation begins with the ansatz $\psi=\mathrm{e}^{i S / \hbar}$ where the action $(S)$ is taken to be complex, and the quantum force is obtained by writing a hierarchy of equations of motion for the phase partial derivatives. We demonstrate how different choices of the trajectory velocity field yield different formulations such as DPM, BOMCA, and ZEVCA. The new derivation is used for two purposes. First, it serves as a common basis for comparing the role of the quantum force in the DPM and BOMCA formulations. Second, we use the new derivation to show that superposing the contributions of real, crossing trajectories yields a nodal pattern essentially identical to that of the exact quantum wavefunction. The latter result suggests a promising new approach to deal with the challenging problem of nodes in Bohmian mechanics.
\end{abstract}

\section{Introduction}

The contrast between the inherent nonlocality of quantum mechanics and the locality of classical mechanics has driven a decades-long quest for a trajectory-based formulation of quantum theory that is exact. In the 1950s, David Bohm, building on earlier work by Madelung ${ }^{1}$ and de Broglie, ${ }^{2}$ developed an exact formulation of quantum mechanics in which trajectories evolve in the presence of the usual Newtonian force plus an additional quantum force. ${ }^{3,4}$ Bohm's formulation was originally developed as an interpretational tool to recover a notion of causality in quantum mechanics. In 1999 Lopreore and Wyatt ${ }^{5}$ demonstrated that the Bohmian formulation can also be used a numerical tool to do quantum calculations. This innovation has coincided well with the ongoing interest of the chemical physics community in finding effective numerical tools for performing multidimensional quantum calculations. The apparently local dynamics of the Bohmian trajectories suggest the possibility of computational advantages compared to fixed grid methods or direct-product basis set methods that scale exponentially with dimensionality. The Lopreore and Wyatt paper has motivated the development of a variety of new numerical approaches for implementing Bohmian mechanics. ${ }^{6-12}$ Reference 13 gives an excellent account of the progress in Bohmian related formulations in recent years.

The Bohmian formulation has two main limitations that currently limit its usefulness as a numerical tool. First, note that the nonlocality of quantum mechanics does not disappear in the Bohmian formulation; it manifests itself in the quantum force term, which needs to be calculated to propagate the quantum trajectories. The quantum force is numerically unstable when the wavefunction is oscillatory. This is related to the second limitation: the quantum force diverges at nodes of the wave-

\footnotetext{
† Part of the special issue "Robert E. Ywatt Festschrift".

* To whom correspondence should be addressed. E-mail: yair.goldfarb@weizmann.ac.il.

$\doteqdot$ Department of Chemical Physics.

$\S$ Department of Mathematics.

"On sabbatical leave from the Department of Mathematics, Bar-Ilan University, Ramat Gan 52900, Israel.
}

function, resulting in the breakdown of the Bohmian formulation in the vicinity of nodes. Indeed, most of the new contributions to Bohmian methodology have been aimed at overcoming these two limitations of the Bohmian formulation.

Among the approaches that have been developed to deal with the calculation of the quantum force are the derivative propagation method (DPM) ${ }^{14}$ and Bohmian mechanics with complex action (BOMCA). ${ }^{15,16}$ The two approaches use a similar procedure, writing a hierarchy of equations of motion for partial derivatives of the phase. The difference between the methods lies in the use of a complex action in BOMCA, as opposed to a real amplitude and phase in DPM. This difference has farreaching consequences that we explore in this paper.

In the first part of this publication we present a unified and compact derivation of Bohmian methods that serves as a common starting point for the DPM, BOMCA, and the zerovelocity complex action (ZEVCA) methods. The derivation is similar to the derivation of BOMCA, but it leaves the choice of the trajectory velocity field undetermined. We demonstrate how different choices of the velocity field yield different formulations, such as DPM, BOMCA, and others. The unified derivation allows for ready comparison of DPM and BOMCA and the role that the quantum force plays in both formulations. In the second part of this paper we use the new derivation to show that superposing the contributions of real, crossing trajectories yields an interference pattern. This contrasts with a cardinal principle of the exact Bohmian formulation: trajectories are not allowed to cross in configuration space. However, because we are dealing with an approximation to Bohmian mechanics, the no-crossing role does not apply. Superposing the contribution from real crossing trajectories represents a promising new avenue for dealing with the challenging problem of nodes in Bohmian mechanics.

This paper is organized as follows. In Section II we present the unified derivation. Section III is dedicated to several special cases of the derivation: ZEVCA (IIIA), BOMCA (IIIB), and DPM (IIIC). In Section IV we show how a variation of the derivation leads to an accurate description of interference and nodal patterns. Section V is a summary and concluding remarks. 


\section{Unified Derivation of Bohmian Methods}

We start by inserting the ansatz (eq 2.1) 17-19 $^{17}$

$$
\psi(x, t)=\exp \left[\frac{i}{\hbar} S(x, t)\right]
$$

into the time-dependent Schrödinger equation (TDSE) (eq 2.2),

$$
i \hbar \psi_{t}=-\frac{\hbar^{2}}{2 m} \psi_{x x}+V(x, t) \psi
$$

where $S(x, t)$ is a complex function, $\hbar$ is Planck's constant divided by $2 \pi, m$ is the mass of the particle, and $V(x, t)$ is the potential energy function. The subscripts denote partial derivatives. The result is a quantum complex Hamilton-Jacobi (HJ) equation (eq 2.3), ${ }^{17-19}$

$$
S_{t}+\frac{1}{2 m} S_{x}^{2}+V=\frac{i \hbar}{2 m} S_{x x}
$$

where we recognize on the left-hand side (LHS) a complex version of the classical HJ equation. On the right-hand side (RHS) is an additional nonclassical term that we refer to as the "complex quantum potential". This term is different from the quantum potential in the conventional Bohmian formulation. The time-independent version of ansatz (2.1) is familiar as the starting point of the Wentzel-Kramers-Brillouin (WKB) approximation. ${ }^{20-22}$ Equation 2.3 was derived by Pauli ${ }^{17}$ as a first step in the WKB derivation. More recently, eq 2.3 was rediscovered by Leacock and Padgett, ${ }^{23}$ who also reverted to a time-independent formulation to calculate eigenvalues. The equation has also been used by several other authors ${ }^{24-26}$ as an analytical tool but not as a constructive method to solve the TDSE with trajectories.

The classical HJ equation can be conveniently solved by integrating along trajectories that satisfy the classical equations of motion. From a mathematical point of view, these trajectories are the characteristics of the classical HJ equation. Here we use an analogous approach to solve the quantum complex HJ equation, by integrating along some family of trajectories. We define a family of trajectories by choosing a velocity field $v(x$, $t$ ), which can be done in an infinite number of ways. (As opposed to the classical case, the velocity field is not necessarily predetermined by the partial differential equation (PDE) we are trying to solve.) Solutions of eq 2.4

$$
\frac{\mathrm{d} x}{\mathrm{~d} t}=v(x, t)
$$

determine trajectories and are parametrized by their initial position $x(0)$. The change of the phase along the trajectory is given by operating on $S(x, t)$ with the Lagrangian time derivative (eq 2.5),

$$
\frac{\mathrm{d}}{\mathrm{d} t} \equiv \frac{\partial}{\partial t}+\frac{\mathrm{d} x}{\mathrm{~d} t} \frac{\partial}{\partial x}=\frac{\partial}{\partial t}+v \frac{\partial}{\partial x}
$$

with the result given by eq 2.6 ,

$$
\frac{\mathrm{d} S}{\mathrm{~d} t}=S_{t}+v S_{x}=\frac{i \hbar}{2 m} S_{x x}-\frac{1}{2 m} S_{x}^{2}-V+v S_{x}
$$

where we have used eq 2.3. The integration of eq 2.6 requires $S_{x}[x(t), t]$ and $S_{x x}[x(t), t]$ (i.e., the values of the first two spatial derivatives of $S$ along the trajectory). Fortunately, it is possible to write equations describing the evolution of the spatial derivatives of $S$. Writing eq 2.7

$$
\left.S_{n}[x(t), t] \equiv \frac{\partial^{n} S}{\partial x^{n}}\right|_{[x(t), t]}
$$

and taking the $n$th spatial derivative of eq 2.3 , we have eq 2.8.

$$
\left(S_{t}\right)_{n}+\frac{1}{2 m}\left(S_{1}^{2}\right)_{n}+V_{n}=\frac{i \hbar}{2 m} S_{n+2}
$$

Inserting the result in the definition of the Lagrangian time derivative of $S_{n}$ gives eq 2.9 .

$$
\begin{array}{r}
\frac{\mathrm{d} S_{n}}{\mathrm{~d} t}=\left(S_{t}\right)_{n}+v S_{n+1}=-\frac{1}{2 m}\left(S_{1}{ }^{2}\right)_{n}-V_{n}+v S_{n+1}+ \\
\frac{i \hbar}{2 m} S_{n+2}
\end{array}
$$

From this procedure we obtain an infinite set of coupled equations describing the evolution of $S$ and its spatial derivatives along a trajectory, namely, eq 2.9 for $n=0,1,2, \ldots$. We note that evaluation of $S_{n}$ requires knowledge of $S_{n+2}$ and $S_{n+1}$ (the latter through the term $\left.\left(S_{1}^{2}\right)_{n}=\sum_{j=0}^{n}\left(\begin{array}{c}n \\ j\end{array}\right) S_{j+1} S_{n-j+1}\right)$. The fact that evaluation of $S_{0}=S$ requires knowledge of $S_{2}=S_{x x}$ expresses the nonlocality of the Schrödinger equation. However, from a formal point of view, integration of the infinite hierarchy of eq 2.9 along the trajectories defined by eq 2.4 can be regarded as a local method of solution of eq 2.3 in the sense that information propagates along individual trajectories independently. This does not contradict the nonlocality of the Schrödinger equation, because not only is the value of $S$ being propagated down a trajectory, but also all its spatial derivatives $\left(S_{1}, S_{2}, \ldots\right)$ are being propagated.

A numerical approximation for solving eq 2.3 can be obtained by truncating the set of eq 2.9 at some $n=N$, by setting $S_{N+1}$ $=S_{N+2}=0$. We summarize the equations of motion of the approximation (eqs 2.10-12);

$$
\begin{gathered}
\frac{\mathrm{d} x}{\mathrm{~d} t}=v[x(t), t] \\
\frac{\mathrm{d} S_{n}}{\mathrm{~d} t}=-\frac{1}{2 m}\left(S_{1}{ }^{2}\right)_{n}-V_{n}+v S_{n+1}+\frac{i \hbar}{2 m} S_{n+2} \\
S_{N+1}=S_{N+2}=0
\end{gathered}
$$

where we emphasize that the solution is given along an individual trajectory $x(t)$ determined by the velocity field $v(x$, $t$ ). The initial conditions of eq 2.11 are given by eq 2.13 ;

$$
S_{n}[x(0), 0]=-\left.i \hbar \frac{\partial^{n} \ln [\psi(x, 0)]}{\partial x^{n}}\right|_{x(0)}
$$

where we have used the relation $S(x, 0)=-i \hbar \ln [\psi(x, 0)]$ from ansatz (eq 2.1). The wavefunction at time $t_{\mathrm{f}}$ at the final position $x\left(t_{\mathrm{f}}\right)$ is given by eq 2.14 .

$$
\psi\left[x\left(t_{\mathrm{f}}\right), t_{\mathrm{f}}\right]=\exp \left\{\frac{i}{\hbar} S_{0}\left[x\left(t_{\mathrm{f}}\right), t_{\mathrm{f}}\right]\right\}
$$

Equations 2.10-12 provide a common starting point for deriving several quantum trajectory methods such as DPM, BOMCA, and ZEVCA, as well as adaptive grid techniques. These methods differ from each other by the specific choice of the velocity field. For the sake of simplicity we present and compare the different methods for $N=2$. For this case, eqs 2.10-12 yield four equations of motion. 


$$
\begin{gathered}
\frac{\mathrm{d} x}{\mathrm{~d} t}=v \\
\frac{\mathrm{d} S_{0}}{\mathrm{~d} t}=-\frac{S_{1}^{2}}{2 m}-V+v S_{1}+\frac{i \hbar}{2 m} S_{2} \\
\frac{\mathrm{d} S_{1}}{\mathrm{~d} t}=-\frac{S_{1}}{m} S_{2}-V_{1}+v S_{2} \\
\frac{\mathrm{d} S_{2}}{\mathrm{~d} t}=-\frac{S_{2}{ }^{2}}{m}-V_{2}
\end{gathered}
$$

The $N=2$ case is unique for two reasons. First, it is the lowest order of truncation for which the equation of motion for the phase $S_{0}$ (eq 2.11 for $n=0$ ) includes a quantum potential term $\left(i \hbar / 2 m S_{2}\right)$. Second, eqs $2.15 \mathrm{a}-\mathrm{d}$ for BOMCA, ZEVCA, and DPM yields the exact solution for an initial Gaussian wavepacket propagating in a potential with up to quadratic terms.

\section{Specific Choices of Trajectory Velocity Fields}

A. ZEVCA. The simplest choice of the velocity field is eq 3.1 .

$$
v(x, t)=0 \Longrightarrow x(t)=x(0)
$$

The resulting trajectories are straight lines; hence, we refer to the resulting approximation as the $\mathrm{ZEVCA}^{27}$ method. The ZEVCA formulation can be regarded as a hybrid between a grid method and a local semiclassical method. In ref 13 section 7.2, Wyatt considers the solution of the global hydrodynamic equations of quantum mechanics on fixed grid points (Eulerian grid) but dismisses its usefulness as a numerical tool. In ref 27, the ZEVCA formulation is shown to produce useful output from local propagation at a single grid point. In this paper we focus on the relation between BOMCA and DPM; hence, we will not elaborate further here on the ZEVCA method. The interested reader is referred to ref 27 .

B. BOMCA. In the BOMCA method, the velocity of the trajectories is set as shown in eq 3.2.

$$
v[x(t), t]=\frac{S_{1}[x(t), t]}{m}
$$

The rationale for this choice is evident if we recall that $S_{1}$ has units of momentum and that $S_{0}$ can be associated with a quantum action field (eq 2.3). Inserting eq 3.2 into eqs $2.15 \mathrm{a}-\mathrm{d}$ yields the $N=2$ equations of motion of BOMCA (eq 3.3).

$$
\begin{gathered}
\frac{\mathrm{d} x}{\mathrm{~d} t}=\frac{S_{1}}{m} \\
\frac{\mathrm{d} S_{0}}{\mathrm{~d} t}=\frac{S_{1}^{2}}{2 m}-V+\frac{i \hbar}{2 m} S_{2} \\
\frac{\mathrm{d} S_{1}}{\mathrm{~d} t}=-V_{1} \\
\frac{\mathrm{d} S_{2}}{\mathrm{~d} t}=-V_{2}-\frac{S_{2}^{2}}{m}
\end{gathered}
$$

The BOMCA formulation was originally presented in terms of equations of motion for partial derivatives of the velocity instead of the phase. ${ }^{15}$ The transformation between the two formulations is straightforward by using the relation in eq 3.2.

Equations 3.3a-d have several attractive features; (1) eqs 3.3a,c are Newton's second law of motion in disguise, resulting in characteristics that are classical trajectories. In other words, the $N=2$ BOMCA approximation does not incorporate a quantum force in computing the trajectories. Note, however, that generally the classical trajectories take on complex values of position and momentum. This results from the complex initial momentum that emerges from eq 2.13 for $n=1$. (2) By recognizing the RHS of eq $3.3 \mathrm{~b}$ as a "quantum Lagrangian", this equation has the familiar structure of the equation of motion for the classical action, with the addition of the quantum potential. The quantum potential is the only $\hbar$-dependent term. Hence, this term is entirely responsible for incorporating the quantum effects in the $N=2$ BOMCA approximation. Note that for $N=3$, a quantum force term appears in the trajectory equations (specifically eq $3.3 \mathrm{c}$ ), yielding complex quantum trajectories.

Equations $3.3 \mathrm{a}-\mathrm{d}$ appear in the context of other timedependent semiclassical methods that use complex classical trajectories. The first of these is the generalized Gaussian wavepacket dynamics (GGWPD) of Huber and Heller. ${ }^{28,29}$ The two main advantages of the BOMCA formulation over GGWPD is that (1) the latter does not provide correction terms to the $N$ $=2$ approximation and (2) the latter cannot be generalized to a non-Gaussian initial wavefunction. The second context in which eqs $3.3 \mathrm{a}-\mathrm{d}$ appear is in the first-order formulation of the complex trajectory method (CTM) of Boiron and Lombardi. ${ }^{30,32}$ This formulation is a complex trajectory version of time-dependent WKB. Although the CTM method is identical to BOMCA for $N=2$, it differs at higher orders of $N$.

The propagation of complex trajectories requires the analytical continuation of the initial wavefunction to the complex plane, as well as a method to reconstruct the wavefunction on the real axis at the desired final time. Regarding the latter, in ref 15 we describe an algorithm to calculate complex initial positions that end at a final time $t_{\mathrm{f}}$ on the real axis $\left(\mathscr{F}\left[x\left(t_{\mathrm{f}}\right)\right]=0\right)$ ). Without going into the details of the algorithm, it is sufficient it to say that it exploits the local analyticity of the mapping $x(0) \rightarrow x\left(t_{\mathrm{f}}\right)$. By inserting the phase $S_{0}$ that corresponds to these trajectories into the original ansatz (eq 2.1), we obtain the wavefunction at a real position $x\left(t_{\mathrm{f}}\right)$ at time $t_{\mathrm{f}}$.

C. DPM. We present here a simplified derivation of the DPM method that yields an equivalent but more compact set of equations than has appeared previously in the literature. ${ }^{13}$ Setting the velocity field to that shown in eq 3.4

$$
v[x(t), t]=\frac{\mathscr{R}\left\{S_{1}[x(t), t]\right\}}{m}
$$

and inserting eq 3.4 into eqs $2.15 \mathrm{a}-\mathrm{d}$, yields the $N=2 \mathrm{DPM}$ equations of motion, eqs $3.5 \mathrm{a}-\mathrm{d}$ :

$$
\begin{gathered}
\frac{\mathrm{d} x}{\mathrm{~d} t}=\frac{\mathscr{R}\left(S_{1}\right)}{m} \\
\frac{\mathrm{d} S_{0}}{\mathrm{~d} t}=\frac{\left[\mathscr{R}\left(S_{1}\right)\right]^{2}}{2 m}-V+\frac{i \hbar}{2 m} S_{2}+\frac{\left[\mathscr{A}\left(S_{1}\right)\right]^{2}}{2 m} \\
\left.\frac{\mathrm{d} S_{1}}{\mathrm{~d} t}=-V_{1}-\frac{i S_{2}}{m} \mathscr{A} S_{1}\right) \\
\frac{\mathrm{d} S_{2}}{\mathrm{~d} t}=-V_{2}-\frac{S_{2}^{2}}{m}
\end{gathered}
$$

The trajectories in the DPM propagate on the real axis, removing the need to extrapolate the wavefunction to the real axis, as is required in BOMCA. But the classical structure that was evident 
in BOMCA is no longer present. Comparing eqs 3.3 and 3.5, we see that a quantum force term appears in the equation of motion for the momentum $S_{1}$ (eq $3.5 \mathrm{c}$ ), and the equation of motion for the phase $S_{0}$ (eq 3.5b) has an "extra" quantum potential term $\left(\left[\mathscr{F}\left(S_{1}\right)\right]^{2} / 2 m\right)$.

Equations $3.5 \mathrm{a}-\mathrm{d}$ are equivalent to those of the DPM as they appear in the literature, ${ }^{13,14}$ as we now show. In the DPM, the wavefunction is represented by the ansatz (eq 3.6);

$$
\psi(x, t)=\exp \left[c(x, t)+\frac{i}{\hbar} s(x, t)\right]
$$

where $s(x, t)$ and $c(x, t)$ are real functions (ansatz 3.6 is essentially identical to the conventional Bohmian ansatz $\psi(x$, $t)=A(x, t) \exp [i / \hbar s(x, t)]$ if one identifies $A(x, t)=\exp [c(x$, $t)])$. Equating eq 3.6 with eq 2.1 yields eq 3.7 .

$$
S(x, t)=s(x, t)-i \hbar c(x, t)
$$

Inserting eq 3.7 into eqs $3.5 \mathrm{a}-\mathrm{d}$ and dividing the results into their real and imaginary parts yields eqs $3.8 \mathrm{a}-\mathrm{g}$.

$$
\begin{gathered}
\frac{\mathrm{d} x}{\mathrm{~d} t}=\frac{s_{1}}{m} \\
\frac{\mathrm{d} s_{0}}{\mathrm{~d} t}=\frac{s_{1}^{2}}{2 m}-V+\frac{\hbar^{2}}{2 m}\left(c_{2}+c_{1}^{2}\right) \\
\frac{\mathrm{d} s_{1}}{\mathrm{~d} t}=-V_{1}+\frac{\hbar^{2}}{m} c_{1} c_{2} \\
\frac{\mathrm{d} s_{2}}{\mathrm{~d} t}=-\frac{s_{2}{ }^{2}}{m}+\frac{\hbar^{2}}{m} c_{2}^{2}-V_{2} \\
\frac{\mathrm{d} c_{0}}{\mathrm{~d} t}=-\frac{s_{2}}{2 m} \\
\frac{\mathrm{d} c_{1}}{\mathrm{~d} t}=-\frac{c_{1} s_{2}}{m} \\
\frac{\mathrm{d} c_{2}}{\mathrm{~d} t}=-\frac{2 c_{2} s_{2}}{m}
\end{gathered}
$$

These equations are equivalent to eqs $3.5 \mathrm{a}-\mathrm{d}$ and are readily seen to be equal to eq 10.10 of ref 13 by taking $N=2$ and identifying $\dot{x}(t)=s_{1} / m$.

Note that the conventional DPM equations of motion also have a quantum correction to the trajectories at truncating order $N=2$. This can be seen in the equation for $s_{1}$ (eq 3.8c), as well as in the equations for $c_{0}, c_{1}$, and $c_{2}$ (eqs $3.8 \mathrm{e}-\mathrm{g}$ ), which have no classical counterparts. Even though the quantum force term in eq $3.8 \mathrm{c}$ is proportional to $\hbar^{2}$, a simple example demonstrates that this term is not small compared with the classical force. Consider the harmonic oscillator potential $V(x)$ $=1 / 2 m \omega^{2} x^{2}$, where $\omega$ is the angular frequency. The timeevolution of the ground state is eq 3.9;

$$
\psi(x, t)=\exp \left[-\alpha_{0} x^{2}-\frac{i \omega}{2} t+\frac{i \gamma_{0}}{\hbar}\right]
$$

where $\alpha_{0}=m \omega / 2 \hbar$ and $\gamma_{0}=-i \hbar / 4 \ln \left(2 \alpha_{0} / \pi\right)$. We recall that eqs $3.8 \mathrm{a}-\mathrm{g}$ (and eqs $3.3 \mathrm{a}-\mathrm{d}$ ) yield the exact solution for an initial Gaussian wavepacket in a harmonic potential. Comparing eqs 3.6 and 3.9 shows that $s$ is position-independent; hence, $s_{1}$ $=0$. As a result, according to eq 3.8 a the eigen-trajectories are

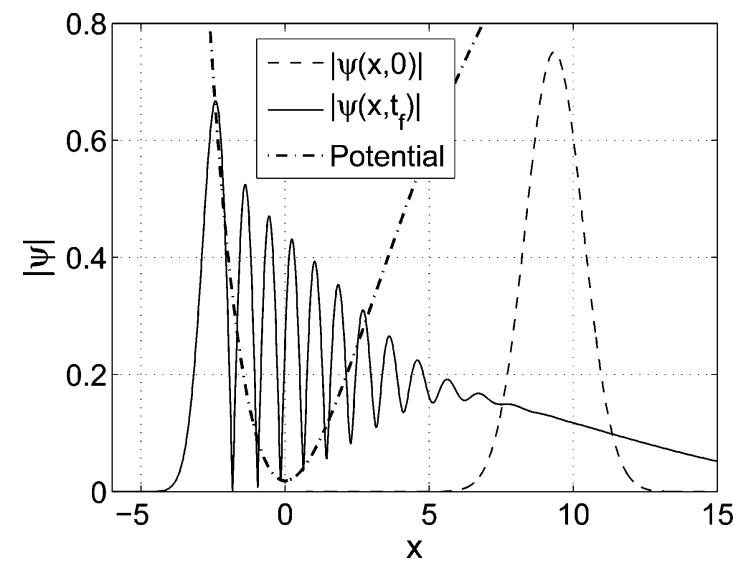

Figure 1. Initial Gaussian wavepacket propagating in a Morse potential. The parameters of the system are given in the text. The final propagation time $\left(t_{\mathrm{f}}\right)$ equals roughly half of the oscillation period of a classical particle located at the center of the initial Gaussian $\left(x_{\mathrm{c}}\right)$. The exact wavefunction was calculated using the split operator method with a Fourier basis.

straight lines (a result that is familiar from the conventional Bohmian formulation). Because $\mathrm{d} s_{1} / \mathrm{d} t=0$, we conclude from eq $3.8 \mathrm{c}$ that the quantum force is equal in its magnitude to the classical force (eq 3.10).

$$
\frac{\hbar^{2}}{m} c_{1} c_{2}=V_{1}[x(0)]=m \omega^{2} x(0)
$$

The LHS of this equation is the quantum force. Not only is the quantum force not negligible, we see from the RHS of eq 3.10 that it depends linearly on the position. The appearance of a significant quantum force in this most "classical" example can be viewed as a result of working with real trajectories. In the BOMCA formulation with its complex trajectories, the quantum force vanishes in this case, which is one of the strongest motivations for studying BOMCA. ${ }^{15}$

\section{Interference with Real Trajectories}

As mentioned in the Introduction, the nodal problem is currently the main obstacle to performing numerical calculations using Bohmian mechanics. In this section we present preliminary results showing that an oscillatory wavefunction in close agreement with the quantum result can be obtained using eqs $2.15 \mathrm{a}-\mathrm{d}$ with real trajectories. Consider an initial Gaussian wavepacket, given by eq 4.1 ,

$$
\psi(x, 0)=\exp \left[-\alpha_{0}\left(x-x_{\mathrm{c}}\right)^{2}+\frac{i}{\hbar} p_{\mathrm{c}}\left(x-x_{\mathrm{c}}\right)+\frac{i}{\hbar} \gamma_{0}\right]
$$

propagating in a Morse potential (eq 4.2).

$$
V(x)=A\left\{[1-\exp (-\beta x)]^{2}-1\right\}
$$

The parameters of the initial Gaussian are $\alpha_{0}=0.5, x_{\mathrm{c}}=9.342$, $p_{\mathrm{c}}=0, \gamma_{0}=-i \hbar / 4 \ln \left(2 \alpha_{0} / \pi\right)$ where we take $m=\hbar=1$ (all quantities are given in atomic units). As for the Morse potential parameters, $A=10.25$ and $\beta=0.2209$. The final propagation time $\left(t_{\mathrm{f}}=5.93\right)$ is chosen so as to produce a strongly oscillating pattern. Figure 1 depicts the potential and wavefunctions.

In both DPM and BOMCA the velocity is taken to be a function of $S_{1}$; in DPM, $v=\mathscr{R}\left(S_{1} / m\right)$, whereas in BOMCA, $v$ $=S_{1} / \mathrm{m}$. We now present an alternative procedure; we take $v$ to be completely independent of $S_{1}$ and set a classical equation of motion for $v$ : 


$$
\frac{\mathrm{d} v}{\mathrm{~d} t}=-\frac{V_{1}[x(t)]}{m}
$$

Equation 4.3 is then supplemented to the set of eqs $2.15 \mathrm{a}-\mathrm{d}$. Furthermore, we extend the freedom in the choice of the velocity field to include the initial conditions for the velocity, which we take to be eq 4.4 .

$$
v[x(0), 0]=\frac{\mathscr{F}\left\{S_{1}[x(0), 0]\right\}}{m}=\frac{2 \alpha_{0}}{m}\left[x(0)-x_{\mathrm{c}}\right]
$$

The term $S_{1}[x(0), 0]$ is obtained by inserting eq 4.1 into eq 2.13 and setting $n=1$. Equation 4.4 defines real initial conditions; taken together with eqs 4.3 and 2.15 a, this yields real classical trajectories (note that if we had taken the initial velocity to be complex $\left(v[x(0), 0]=S_{1}[x(0), 0] / m\right)$ we would have obtained the BOMCA equations, eqs $3.3 \mathrm{a}-\mathrm{g}$ ). The next step is to solve eqs $2.15 \mathrm{a}-\mathrm{d}$ and 4.3 with initial conditions given by eqs 2.13 and 4.4 , respectively, for a series of initial positions $\{x(0)\}$ in the vicinity of $x_{\mathrm{c}}$. The wavefunction at time $t_{\mathrm{f}}$ at final position $x_{\mathrm{f}}$ is given by eq 2.14 , where $S_{0}\left[x\left(t_{\mathrm{f}}\right), t_{\mathrm{f}}\right]$ is the solution of eq $2.15 b$.

In Figure 2 we plot the trajectories obtained. The trajectories can be divided into two overlapping groups that we refer to as branches. The first (reflected) branch (solid lines) is the locus of trajectories that have reached their classical turning point and were reflected by the exponential barrier of the potential. The second branch (dashed lines) is the locus of trajectories that by $t_{\mathrm{f}}$ did not reach their classical turning point. Thus, to an arbitrary final position $x(x \gtrsim-2.8)$, these correspond to two initial positions and two associated trajectories.

The question arises, if two trajectories and, therefore, two values of $S_{0}\left(x, t_{\mathrm{f}}\right)$ correspond to each final position $x$, how should we determine the wavefunction $\psi\left(x, t_{\mathrm{f}}\right)$ ? The clear distinction between the two branches allows us to associate a wavefunction with each branch, which we will call $\psi_{1}\left(x, t_{\mathrm{f}}\right)$ and $\psi_{2}\left(x, t_{\mathrm{f}}\right)$. We apply the superposition principle to reconstruct the wavefunction at $x$, obtaining eq 4.5 .

$$
\psi\left(x, t_{\mathrm{f}}\right)=\psi_{1}\left(x, t_{\mathrm{f}}\right)+\psi_{2}\left(x, t_{\mathrm{f}}\right)
$$

In Figure 3 we compare the exact wavefunction along with $\left|\psi_{1}\left(x, t_{\mathrm{f}}\right)\right|,\left|\psi_{2}\left(x, t_{\mathrm{f}}\right)\right|$, and $\left|\psi\left(x, t_{\mathrm{f}}\right)\right|=\left|\psi_{1}\left(x, t_{\mathrm{f}}\right)+\psi_{2}\left(x, t_{\mathrm{f}}\right)\right|$. For values of $x$ larger than $\approx-2.8$ (the position of the maximum of $\left.\left|\psi\left(x, t_{\mathrm{f}}\right)\right|\right)$, the superposition yields a surprisingly accurate approximation of the oscillating wavefunction; even though the wavefunctions $\psi_{1}\left(x, t_{\mathrm{f}}\right)$ and $\psi_{2}\left(x, t_{\mathrm{f}}\right)$ exhibit no oscillations whatsoever, their superposition yields strong oscillations and a node near the maximum. Note that $\psi_{2}\left(x, t_{\mathrm{f}}\right)$ provides the "main" contribution to the final wavefunction; $\psi_{1}\left(x, t_{\mathrm{f}}\right)$, which originates from the reflected branch, contributes to $\psi\left(x, t_{\mathrm{f}}\right)$ only where the wavefunction oscillates. As the classical turning point is approached, the amplitudes arising from the different branches diverge, and the superposition of contributions appears to have no physical significance.

As noted above, the BOMCA equations for $N=2$ are identical to those of GGWPD. In both cases, the trajectories that are propagated obey classical equations of motion but are complex. In this section we described a modification of BOMCA in which the classical trajectories are taken to be real. It is interesting to speculate if there might be a connection with thawed Gaussian propagation, in which the equations of motion are the same as those for GGWPD, but the trajectories are real. Consistent with this conjecture is the observation that in thawed Gaussian propagation there is no need for a root search, as is

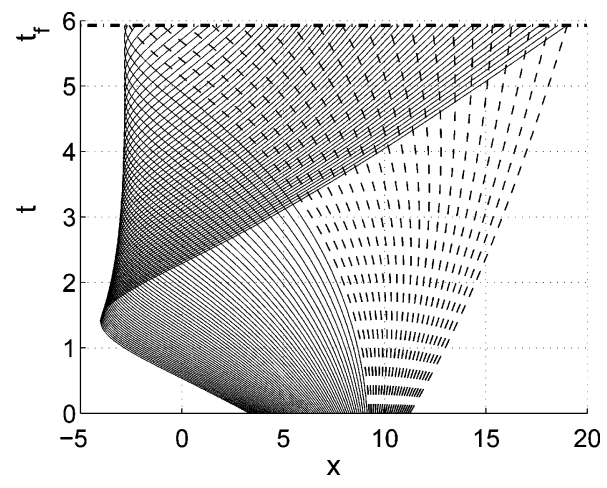

Figure 2. Real classical trajectories obtained by solving Newton's second law of motion (eq 4.3) for a Morse potential (eq 4.2) with initial conditions given by eq 4.4. The parameters of the propagation are given in the text. The final propagation time $\left(t_{\mathrm{f}}\right)$ is marked explicitly. The trajectories can be divided into two overlapping branches. The first branch (solid lines) is the locus of trajectories that have reached their classical turning point and were reflected by the exponential barrier of the potential. The second branch (dashed lines) is the locus of trajectories that by $t_{\mathrm{f}}$ did not reach their classical turning point.

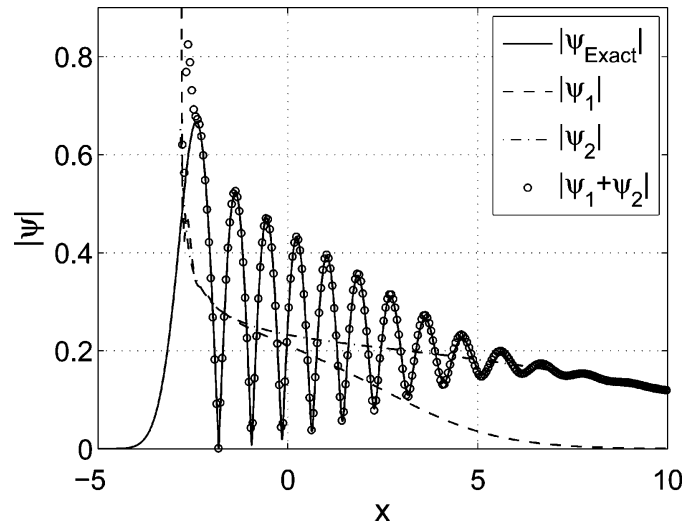

Figure 3. A comparison between the exact wavefunction with the contribution of the first branch $\left|\psi_{1}\left(x, t_{\mathrm{f}}\right)\right|$, the second branch $\left|\psi_{2}\left(x, t_{\mathrm{f}}\right)\right|$, and a superposition of both $\left|\psi\left(x, t_{\mathrm{f}}\right)\right|=\left|\psi_{1}\left(x, t_{\mathrm{f}}\right)+\psi_{2}\left(x, t_{\mathrm{f}}\right)\right|$. For $x \gtrsim$ -2.8 (the position of the maximum of the exact wavefunction) the superposition procedure yields a surprisingly accurate approximation of the oscillating wavefunction, even though the wavefunctions $\mid \psi_{1}(x$, $\left.t_{\mathrm{f}}\right) \mid$ and $\left|\psi_{2}\left(x, t_{\mathrm{f}}\right)\right|$ exhibit no oscillations whatsoever. The procedure is undefined in the classically forbidden region, which the classical trajectories cannot access. The exact wavefunction was calculated using the split operator method with a Fourier basis.

true in the real-trajectory version of BOMCA. However, the correspondence cannot be exact. In the real-trajectory version of BOMCA, in principle, every point in coordinate space is propagated; however, in thawed Gaussian propagation the initial wavefunction is decomposed into Gaussians, and any decomposition that satisfies completeness is allowed. Moreover, in real-trajectory BOMCA the trajectories have no width and no functional form, whereas in thawed Gaussian propagation there is always some residual signature of the Gaussian decomposition. For example, Gaussians that reach the turning point have part of their amplitude extending into the classically forbidden region, whereas in real-trajectory BOMCA such penetration into the classically forbidden region is absent.

\section{Summary}

We have presented a unified and compact derivation of Bohmian methods that serves as a common starting point for several approximations. In particular, the new approach was used to derive and compare the DPM, BOMCA, and ZEVCA 
methods. We focused on the role of the quantum force in the DPM and BOMCA methods and showed that for $N=2$, the lowest order of truncation for which a quantum potential is present, the BOMCA formulation is closer to the classical equations of motion than DPM, although the trajectories are complex. We also demonstrated that an interference pattern can be obtained by superposing the contributions from real classical trajectories into what is otherwise essentially a Bohmian formulation.

Because nodes in quantum mechanics arise from interfering amplitudes, it is only natural to attempt to solve the nodal problem in Bohmian mechanics by applying the superposition principle. This suggests decomposing the wavefunction into two nodeless parts and propagating each part separately using trajectories. ${ }^{11,31}$ However, because nodeless wavefunctions do not generally remain nodeless, this approach generally requires a series of time-dependent decompositions of the total wavefunction. Such decompositions are somewhat arbitrary and are often valid only for relatively short times. In the scheme we presented, an oscillatory wavefunction is successfully decomposed for all times considered into two nodeless, nonoscillatory parts $\left(\psi_{1}\right.$ and $\left.\psi_{2}\right)$. As such, the scheme represents a promising new avenue for dealing with the challenging problem of nodes in Bohmian mechanics. We are currently exploring an analogous idea of applying the superposition principle to complex crossing trajectories in $\mathrm{BOMCA}^{16}$ and in time-dependent $\mathrm{WKB} ;{ }^{32}$ the results are very promising and will be published elsewhere. In addition, although we do not yet have final results, it seems that path integral techniques can provide a rigorous justification of the superposition principle in Bohmian methods and the limits of its applications.

Acknowledgment. This work was supported by the Israel Science Foundation (No. 576/04).

\section{References and Notes}

(1) Madelung, E. Z. Z. Phys. 1926, 40, 322.

(2) de Broglie, L. C. R. Acad. Sci. (Paris) 1926, 183, 447.

(3) (a) Bohm, D. Phys. Rev. 1952, 85, 166. (b) Bohm, D. Phys. Rev. 1952, 85,180 .
(4) Bohm, D.; Hiley, B. J. The Undivided Universe: An Ontological Interpretation of Quantum Theory; Routledge: London, 1993.

(5) Lopreore, C. L.; Wyatt, R. E. Phys. Rev. Lett. 1999, 82, 5190.

(6) Liu, J.; Makri, N. J. Phys. Chem. A 2004, 108, 5408-5416.

(7) Bitter, E. R.; Wyatt, R. E. J. Chem. Phys. 2000, 113, 8888. 567.

(8) Garashchuk, S.; Rassolov, V. A. Chem. Phys. Lett. 2002, 364, 562-

(9) Burghardt, I.; Cederbaum, L. S. J. Chem. Phys. 2002, 115, 10303.

(10) Gindensperger, E.; Meier, C.; Beswick, J. A. J. Chem. Phys. 2000, $113,9369$.

(11) Poirier, B. J. Chem. Phys. 2004, 121, 4501.

(12) Kendrick, B. K. J. Chem. Phys. 2003, 119, 5805.

(13) Wyatt, R. E. Quantum Dynamics with Trajectories: Introduction to Quantum Hydrodynamics; Springer: New York, 2005.

(14) Trahan, C. J.; Hughes, K.; Wyatt, R. E. J. Chem. Phys. 2003, 118, 9911.

(15) Goldfarb, Y.; Degani, I.; Tannor, D. J. J. Chem. Phys. 2006, 125, 231103.

(16) (a) Goldfarb, Y.; Tannor, D. J. J. Chem. Phys. submitted. (b) Goldfarb, Y.; Tannor, D. J. arXiv.org [Online] 2007, arXiv:0706.3507v1.

(17) Pauli, W. Die allgemeine Prinzipien der Wellenmechanik, Handbuch der Physik, 2nd ed; Part 1; Springer-Verlag: Berlin, 1933; Vol. 24.

(18) Gottfried, K. Quantum Mechanics: Foundations; W. A. Benjamin: New York, 1966; Vol 1.

(19) Tannor, D. J. Introduction to Quantum Mechanics: A Time Dependent Perspective; University Science Press: Sausalito, 2006.

(20) Wentzel, G. Z. Phys. 1926, 38, 518.

(21) Kramers, H. A. Z. Phys. 1926, 39, 828.

(22) (a) Brillouin, L. C. R. Acad. Sci (Paris) 1926, 183, 24. (b) Brillouin, L. J. Phys. 1926, 7, 353

(23) Leacock, R. A.; Padgett, M. J. Phys. Rev. D 1983, 28, 2491.

(24) John, M. V. Found. Phys. Lett. 2002, 15, 329.

(25) (a) Yang, C. D. Ann. Phys. 2005, 319, 399. (b) Yang, C. D. Ann. Phys. 2005, 319, 444. (c) Yang, C. D. Int. J. Quantum Chem. 2006, 106, 1620 .

(26) Sanz, A. S.; Borondo, F.; Miret-Artés, S. J. Phys.:Condens. Matter 2002, 14, 6109 .

(27) (a) Goldfarb, Y.; Degani, I.; Tannor, D. J. Chem. Phys. accepted 2007. (b) Goldfarb, Y.; Degani, I.; Tannor, D. J. arXiv.org [Online] 2007, arXiv:0705.2132v1.

(28) Huber, D.; Heller, E. J. J. Chem. Phys. 1987, 87, 5302.

(29) Huber, D.; Heller, E. J.; Littlejohn, R. G. J. Chem. Phys. 1988, 89, 2003.

(30) Boiron, M.; Lombardi, M. J. Chem. Phys. 1998, 108, 3431.

(31) Babyuk, D.; Wyatt, R. E. J. Chem. Phys. 2004, 121, 9230.

(32) (a) Goldfarb, Y.; Schiff, J.; Tannor, D. J. J. Chem. Phys. submitted. (b) Goldfarb, Y.; Schiff, J.; Tannor, D. J. arXiv.org [Online] 2007, arXiv: $0707.0117 \mathrm{v} 1$. 